\title{
miR-380-3p regulates melanogenesis by targeting SOX6 in melanocytes from alpacas (Vicugna pacos)
}

\author{
Xuexian Liu', Bin Du', Pengqian Zhang ${ }^{2}$, Junzhen Zhang ${ }^{1}$, Zhiwei Zhu ${ }^{3}$, Bo Liu and Ruiwen Fan ${ }^{1 *}$ (D)
}

\begin{abstract}
Background: Melanocytes are derived from neural crest stem cells in the embryonic stage. In mature melanocytes, a series of complex enzyme-catalyzed reactions leads to the production of melanins, which determine the hair and skin colors of animals. The process of melanogenesis is complex and can be regulated by mRNA, microRNAs (miRNAs) and long noncoding RNAs (IncRNAs) genes. MiRNAs are a type of endogenous noncoding RNA approximately $22 \mathrm{nt}$ in size that predominantly regulate gene expression by inhibiting translation. miR-380-3p is a candidate miRNA potentially related to melanogenesis. To better understand the mechanism of miR-380-3p melanogenesis regulation, plasmids to overexpress or knockdown miR-380-3p were transfected into alpaca melanocytes, and their effects on melanogenesis were evaluated.

Results: In situ hybridization identified a positive miR-380-3p signal in alpaca melanocyte cytoplasm. Luciferase activity assays confirmed that SOX6 is targeted by miR-380-3p. miR-380-3p overexpression and knockdown in alpaca melanocytes respectively downregulated and upregulated SOX6 expression at the mRNA and protein levels. Additionally, miR-380-3p overexpression and knockdown, respectively, in alpaca melanocytes decreased and increased the mRNA levels of melanin transfer-related genes, including microphthalmia-associated transcription factor (MITF), tyrosinase (TYR), tyrosine-related protein-1 (TYRP1), and dopachrome tautomerase (DCT). In contrast, miR-380-3p overexpression and knockdown respectively increased and decreased the mRNA levels of $\beta$-catenin. Additionally, the effect of miR-380-3p on melanogenesis was assessed by Masson-Fontana melanin staining.

Conclusions: The results demonstrated that miR-380-3p targeted SOX6 to regulate melanogenesis by influencing $\beta$ catenin and MITF transcription and translation, which reduced the expression of downstream genes, including TYR, TYRP1, and DCT. These results provide insights into the mechanisms through which miR-380-3p controls melanogenesis.
\end{abstract}

Keywords: microRNA-380-3p, Sex-determining region Y-box 6, Melanogenesis, Microphthalmia-associated transcription factor, Tyrosinase, Tyrosine-related protein-1, Dopachrome tautomerase

\section{Background}

Alpaca (Vicugna pacos) is a fiber-producing camelid with 22 coats of hair determined by genetic and endocrine factors [1]. An increasing number of genes have been reported to influence mammalian hair color formation. Microphthalmiaassociated transcription factor (MITF) is one of these genes and is an important transcription factor that regulates the key enzymes of melanin production, including tyrosinase (TYR), tyrosinase-related protein 1 (TYRP1) and

\footnotetext{
*Correspondence: ruiwenfan@163.com

${ }^{1}$ College of Animal Science and Veterinary Medicine, Shanxi Agricultural

University, Mingxian South Road, Taigu, China

Full list of author information is available at the end of the article
}

dopachrome tautomerase (DCT) [2]. Moreover, MITF is involved in several pathways that regulate melanogenesis via mRNA, miRNA and lncRNA encoding genes.

miRNAs are essential regulators of cell proliferation and differentiation during development. miRNA biogenesis involves precursor miRNA cleavage into mature miRNAs in a process catalyzed by endonucleases such as Dicer [3]. miRNAs control a wide range of biological processes, including development, differentiation, proliferation and stress response [4]. Numerous miRNAs have been reported to be involved in the regulation of melanogenesis [5], and these miRNAs include miR-137, miR-434-5p, miR-218, miR-21a-5p, miR-145, miR-340 and miR-27a-3p [6-12].

(c) The Author(s). 2019 Open Access This article is distributed under the terms of the Creative Commons Attribution 4.0 International License (http://creativecommons.org/licenses/by/4.0/), which permits unrestricted use, distribution, and 
We have demonstrated that miR-508-3p [13] and miR-25 [14] regulate melanogenesis by targeting MITF in alpaca melanocytes. miR-143-5p regulates alpaca melanocyte migration, proliferation, and melanogenesis [15]; miR-5110 regulates eumelanin production and transfer in alpaca (Vicugna pacos) melanocytes by cotargeting melanophilin (MLPH) and Wnt family member 1 (Wnt1) [16]; and lpamiR-nov-66 targeting of soluble guanylate cyclase (sGC) regulates melanogenesis via the cyclic adenosine monophosphate (cAMP) pathway [17]. To identify more miRNAs that regulate melanogenesis, we identified a potential melanogenesis regulator, miR-380-3p, by comparing the miRNA expression profiles of sheep skins with different hair colors in a previous experiment (unpublished data). miR-380-3p, also known as miR-380 [18], has been found to be expressed in the brain of mice [19] and is differentially expressed in the medial nasal processes in the fetal tissue between gestational days 10 and 11.5 [20]. This miRNA is not affected by Dicer ablation in Sertoli cells [21], suggesting that it is generated by Dicer-independent miRNA biogenesis pathways, such as the Ago2 pathway [22]. We used a bioinformatic analysis to predict if miR-380-3p may targets the sex-determining region Y-box 6 (SOX6) gene. SOX6 and members of the SOXD group, such as SOX5 and SOX13 [23], act as either positive or negative transcription modulators via various mechanisms [24]. SOX5 and SOX6 are coexpressed with SOXE genes in melanocytes [25]. SOX6 promotes $\beta$-catenin degradation by inhibiting the Wnt signaling pathway in adipogenesis [26]. In addition, Wnt/ $\beta$-catenin signaling is involved in melanogenesis [27]. Therefore, it was deduced that SOX6 may have roles in melanogenesis. We found that SOX6 is expressed and can act as a target for phosphorylation by cyclin-dependent kinase 5 (CDK5) in alpaca melanocytes. Furthermore, SOX6 positively regulates melanogenesis by binding to $\beta$-catenin to regulate cyclin D1 [28]. However, CDK5 regulates melanogenesis and changes hair colors in mice [29]. The present study was undertaken to test the hypothesis that miR-380p might play a role in melanogenesis by targeting SOX6 in alpaca (Vicugna pacos) melanocytes.

\section{Results}

\section{miR-380-3p and SOX6 expression in alpaca skin and} melanocytes

To confirm the relationship between miR-380-3p and hair color, the expression of miR-380-3p was analyzed by quantitative real-time PCR (RT-qPCR) in alpaca skin samples with white, brown and black hair. The results showed that miR-380-3p expression was significantly higher $(P<0.01)$ in alpaca skin samples with white hair than in the skin samples with black hair and brown hair (Fig. 1a). Additionally, we found that SOX6 levels were lower in alpaca skin samples with white hair than in skin samples with black hair and brown hair at the mRNA and protein levels (Fig. 1b-d, $P<0.01$ ). In situ hybridization of miR-380-3p in alpaca melanocytes showed that miR-380-3p is localized in the cytoplasm of these cells, whereas no specific hybridization signal was detected in the cells hybridized with the negative control (NC) probe (Fig. 1e).

\section{miR-380-3p targets the predicted mRNA binding site in the 3'-UTR of SOX6}

The miRBase (http://www.mirbase.org/), miRTarBase (http://www.mirtarbase.mbc.nctu.edu), and miRDB (http://www.mirdb.org/) databases were used to predict the binding site of miR-380-3p in the SOX6 mRNA. The predicted seed sequence of miR-380-3p is conserved in various mammalian species and is complementary to the predicted binding site in the 3 '- untranslated region (UTR) of SOX6 (Fig. 2a). To assess whether miR-380-3p regulates SOX6 at the predicted binding site, luciferase reporter assays were performed in $293 \mathrm{~T}$ cells cotransfected with the miR-380-3p expressing vector and luciferase reporter constructs containing the wild-type SOX6 3'-UTR sequence or mutated binding sites. miR-380-3p overexpression suppressed the reporter signal by approximately $40 \%(P<0.01)$ when the wild-type UTR was used, whereas the luciferase activity did not change when the mutant binding sites were used (Fig. 2b). These data indicate that miR-380-3p binds to and regulates SOX6 in a sequence-specific manner through the predicted 3'-UTR binding site.

\section{Effect of miR-380-3p on SOX6 mRNA and protein expression}

To evaluate whether high levels of miR-380-3p and low levels of SOX6 in alpaca skin with white hair are associated, plasmids expressing miR-380-3p or its inhibitor were transfected into alpaca melanocytes, and SOX6 expression was evaluated. RT-qPCR data indicate a significant increase in miR-380-3p expression in melanocytes transfected with the miRNA expression plasmid compared to that in the cells transfected with the NC plasmid (Fig. 3a, $P<0.001$ ). miR-380-3p overexpression resulted in a significant decrease in SOX6 abundance at the mRNA (Fig. 3b, $P<0.01$ ) and protein levels (Fig. 3c-d, $P<0.01$ ). These data suggest that miR-380-3p targets SOX6.

\section{Effect of miR-380-3p on the expression of $\beta$-catenin and melanogenic genes}

SOX6 has been shown to interact physically with $\beta$ catenin in pancreatic $\beta$-cells [30]. To determine whether SOX6 has similar effects on $\beta$-catenin in melanocytes, $\beta$ catenin mRNA and protein levels were examined in melanocytes transfected with miR-380-3p and inhibitor plasmids. The results showed that $\beta$-catenin expression is significantly upregulated or downregulated at the mRNA 

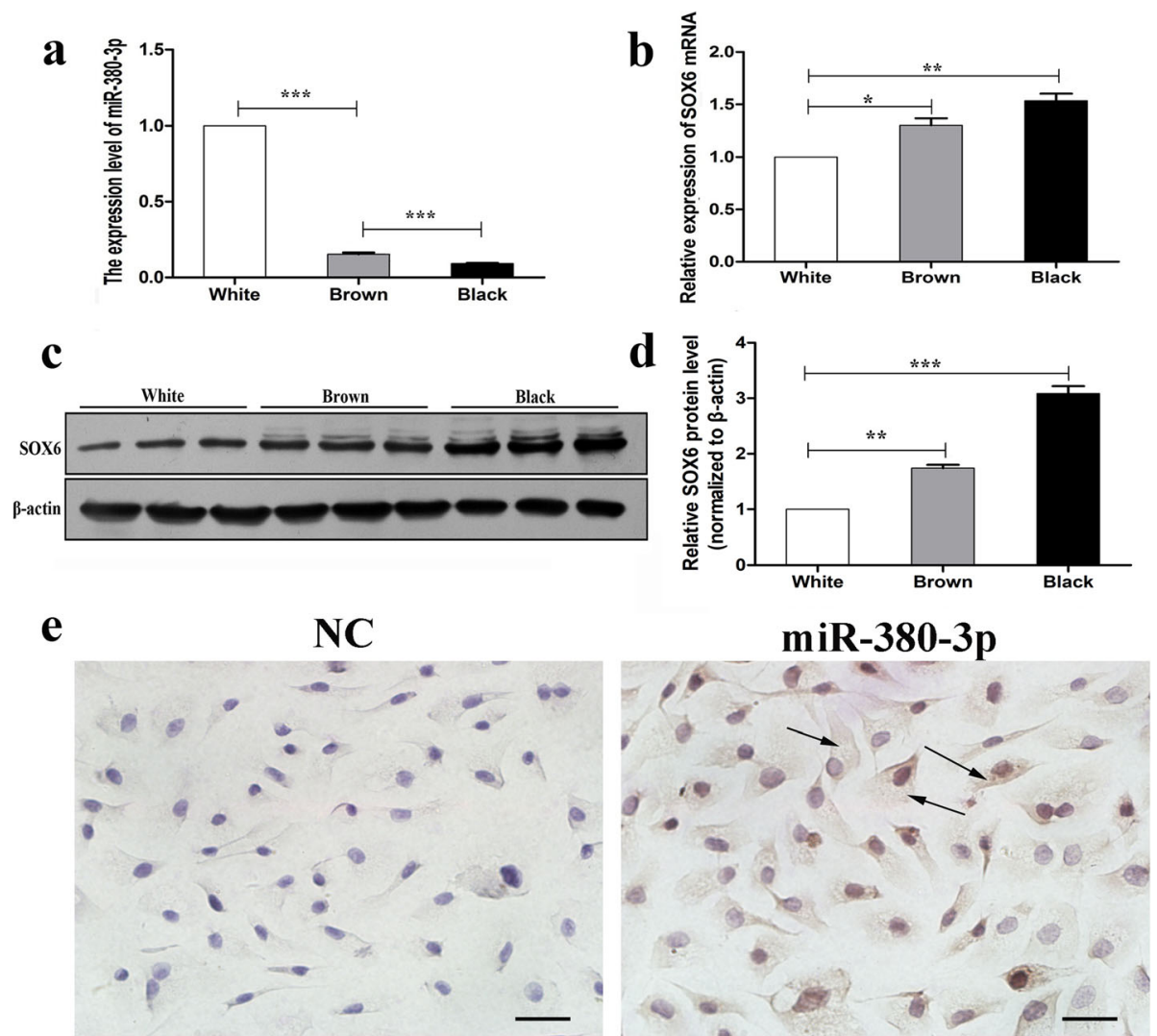

Fig. 1 miR-380-3p and SOX6 expression in alpaca skin samples with different hair colors and in melanocytes. a RT-qPCR analysis of miR-380-3p expression in alpaca skin samples with different hair colors. b RT-qPCR analysis of SOX6 mRNA expression in alpaca skin samples with different hair colors. c Western blot analysis of SOX6 protein expression in alpaca skin samples with different hair colors. d Quantitative analysis of SOX6 protein expression in alpaca skin samples with different hair colors. e Localization of miR-380-3p in alpaca melanocytes in vitro by in situ hybridization analysis. miRNA, mRNA and protein expression levels were normalized to those of U6 mRNA, $18 S$ rRNA and $\beta$-actin, respectively. The bars represent the mean \pm standard error $(n=3) .{ }^{*} P<0.05,{ }^{*} P<0.01,{ }^{* *} P<0.001$

and protein levels by miR-380-3p overexpression or knockdown vectors, respectively. Additionally, the expression levels of typical melanogenic genes, including MITF, TYR, TYRP1, and DCT, were significantly decreased or increased in melanocytes transfected with miR-380-3p overexpression or knockdown vectors, respectively, compared with those in cells transfected with the NC plasmid (Fig. 4a-c, ${ }^{*} P<0.05$, ${ }^{* * *} P<0.01$, **** $P<0.001$ ).

\section{Effect of miR-380-3p on melanin production}

To determine whether miR-380-3p influences melanin production, total alkali-soluble melanin was quantified in alpaca melanocytes transfected with the miR-380-3p expression plasmid and its inhibitor. miR-380-3p overexpression and knockdown in alpaca melanocytes reduced alkali-soluble melanin production by $50 \%$ and increased alkali-soluble melanin production by 1.25 -fold over what was observed in the control group (Fig. 5a, $P<0.001$ ). Next, melanocytes were collected by centrifugation (Fig. 5b). To verify the effect of miR-380-3p, we performed miR-380-3p and SOX6 cotransfection in the melanocytes.
The results showed that miR-380-3p and SOX6 can rescue melanin production (Fig. $5 \mathrm{c}-\mathrm{d}, P<0.001$ ). MassonFontana melanin staining indicated that the number of melanin particles (arrow) was significantly decreased after miR-380-3p overexpression (Fig. 5e, f, $P<0.001$ ).

\section{Discussion}

Melanocytes are melanin-producing cells responsible for skin and hair pigmentation. They contribute to the appearance of the skin and provide protection from ultraviolet radiation damage [31]. Numerous studies have focused on identifying the genes that regulate melanogenesis because of their involvement in melanoma formation. A number of precise pigmentation mechanisms determine the color of the skin or hair of alpacas, which have 22 natural hair colors; microRNAs play important roles in these effects. miR-380-3p is not expressed in normal human melanocytes, but it is expressed in melanoma cell lines [32]. However, our results indicate that miR$380-3 p$ is expressed in normal alpaca melanocytes and is 
a
SOX6 mRNA 5' CGCCUCUCACUACAUC 3'
miR-380-3p

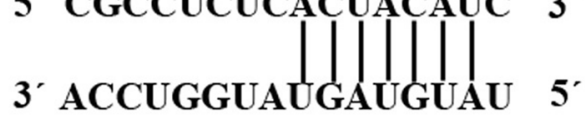
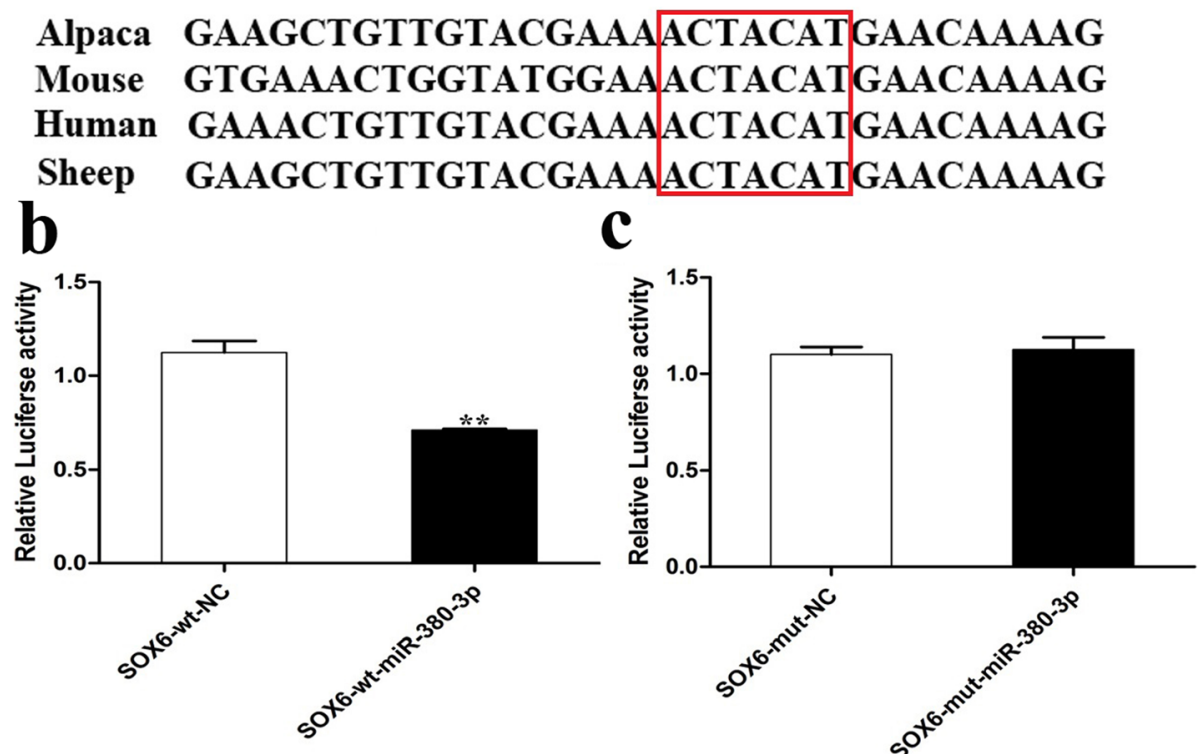

Fig. 2 miRNA-380-3p directly targets SOX6. a miR-380-3p seed sequence and binding site in the SOX6 3' UTR of various mammal species. b, c Luciferase assays in 293T cells cotransfected with miR-380-3p or a negative control (NC) and the luciferase reporter constructs containing either the wild-type SOX6 3'-UTR (SOX6-wt) or a mutated 3'-UTR (SOX6-mut). ${ }^{*} P<0.01$

localized in the cytoplasm, suggesting that miR-380-3p plays a certain role in melanocyte biology in alpacas.

Using bioinformatics tools, we predicted that SOX6 is a target gene of miR-380-3p, suggesting that the interaction between miR-380-3p and SOX6 is conserved in mammals. Additionally, we found that SOX6 is differentially expressed at the mRNA and protein levels in alpaca skin with different hair colors. miRNAs in animals are considered to repress target mRNA expression at the translation level with little or no decrease in mRNA abundance; however, an increasing number of studies have revealed that mRNA destabilization can be promoted by miRNAs via the GW182 protein (TNRC6A-C in mammals and GW182 or Gawky in Drosophila) [33]. This relationship suggests that miR-380-3p can not only repress SOX6 translation but also induce SOX6 mRNA decay. SOX6 is a novel gene regulating melanogenesis, as discovered in our previous study [28]. We have found that SOX6 downregulation leads to an increase in $\beta$-catenin expression and is accompanied by a decrease in cyclin D1 expression in melanocytes [28]. Thus, the effect of miR-380$3 \mathrm{p}$ on $\beta$-catenin expression is apparently mediated by SOX6 via the cyclin $D 1 / \beta$-catenin pathway. Previous studies have shown that the MITF promoter is potently activated by $\beta$ catenin in B16 melanoma cells and melanocytes [34, 35]. It has also been reported that in numerous types of cancer, including melanoma, decreased MITF expression is induced by the $\mathrm{Wnt} / \beta$-catenin pathway [36].

Activation of the $\mathrm{Wnt} / \beta$-catenin pathway stabilizes $\beta$ catenin in the cytoplasm, leading to its translocation into the nucleus where it associates with TCF/LEF transcription factors and activates the expression of MITF gene [37]. All these studies point to a positive relationship between the expression of $\beta$-catenin and MITF. However, our data showed that upregulation of $\beta$-catenin was associated with decreased expression of MITF. We cannot provide a good explanation for this result. However, it is possible that SOX6 may regulate the expression of MITF directly. Indeed, MITF is a direct target gene of SOX10, a member of the SOXD family, which is essential for melanocyte development [38]. MITF is known to regulate the transcription of three genes encoding major pigmentation enzymes: TYR, TYRP1, and DCT [39]. Consequently, MITF downregulation in alpaca melanocytes during miR-380-3p overexpression results in a decrease in TYR, TYRP1, and DCT expression at the mRNA and protein levels, which in turn results in reduced melanin production (Fig. 6).

\section{Conclusions}

Our results demonstrate that miR-380-3p binds to the 3'UTR of SOX6 mRNA, thus downregulating its 


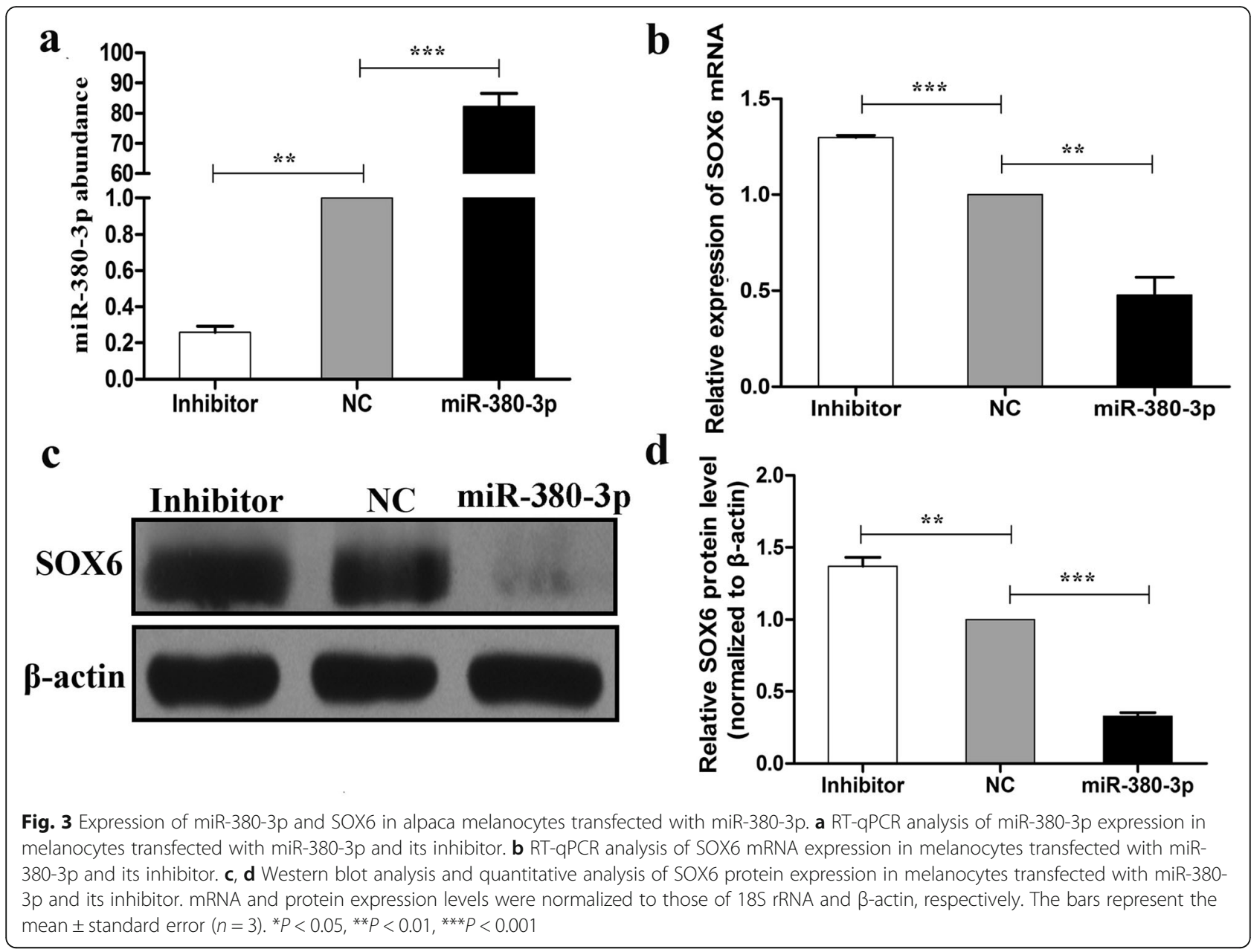

expression at the mRNA and protein levels. The downregulation of SOX6, in turn, affects $\beta$-catenin and MITF transcription and translation, causing a reduction in melanin production mediated by the downstream genes TYR, TYRP1, and DCT. These results indicate that miR380-3p plays an important role in melanogenesis and provide insight into the mechanisms through which miR-380-3p controls melanogenesis by targeting SOX6.

\section{Methods}

\section{In situ hybridization}

Punch skin biopsies $(4 \times 8 \mathrm{~mm})$ were obtained from three healthy male 1-year-old alpacas with white, brown (spectrophotometric assay for alkali-soluble melanins 1.27 1.95 and eumelanin 0.47 0.78) and black hair colors from an alpaca farm of Shanxi Agricultural University. The biopsies were obtained after local anesthesia by subsequent injection of $2 \mathrm{ml}$ of xylazine hydrochloride $(0.05 \mathrm{mg} / \mathrm{ml})$ into the cervical muscles of the alpacas. After $15 \mathrm{~min}, 1 \mathrm{ml}$ of a normal saline solution containing 800,000 units of penicillin and 400,000 units of streptomycin was mixed with $1 \mathrm{ml}$ of $2 \%$ lidocaine $(20 \mathrm{mg} / \mathrm{ml})$ at a ratio of $1: 1$, and $0.5 \mathrm{ml}$ of the mixed solution was then injected at each of three skin sampling sites. The samples were immersed immediately in melanocyte basal medium (Promocell GMBH, Heidelberg, Germany) containing $25 \mathrm{mMN}$-(2-hydroxyethyl)-piperazine- $\mathrm{N}^{\prime}$-(2-ethanesulfonic acid) (HEPES), $400 \mathrm{U} / \mathrm{ml}$ penicillin and $400 \mu \mathrm{g} / \mathrm{ml}$ streptomycin and transported to the laboratory on ice. The alpaca melanocytes were prepared as previously described [40]. miR-380-3p expression in melanocytes was evaluated by in situ hybridization. In detail, melanocytes were treated with proteinase $\mathrm{K}$ (Roche Applied Science, Shanghai, China), washed three times with $0.1 \mathrm{M}$ phosphate-buffered saline (PBS) (Solarbio, Beijing, China) and fixed in $4 \%$ paraformaldehyde for $30 \mathrm{~min}$ on slides. After the cells were prehybridized with $20 \mu \mathrm{l}$ of the prehybridization buffer for $3 \mathrm{~h}, 3 \mathrm{pmol}$ of a digoxigenin-labeled miR-380-3p probe was added to $20 \mu$ of the hybridization buffer to carry out the hybridization at $38^{\circ} \mathrm{C}$ overnight. The slides were washed twice with SSC at $37^{\circ} \mathrm{C}$ and incubated with 1:1000 diluted alkaline phosphatase-conjugated mouse anti-digoxigenin antibody (Roche Applied Science) for $4 \mathrm{~h}$ at $37^{\circ} \mathrm{C}$. Finally, diaminobenzidine (DAB) was used as the substrate for the alkaline phosphatase reaction. The miR- 

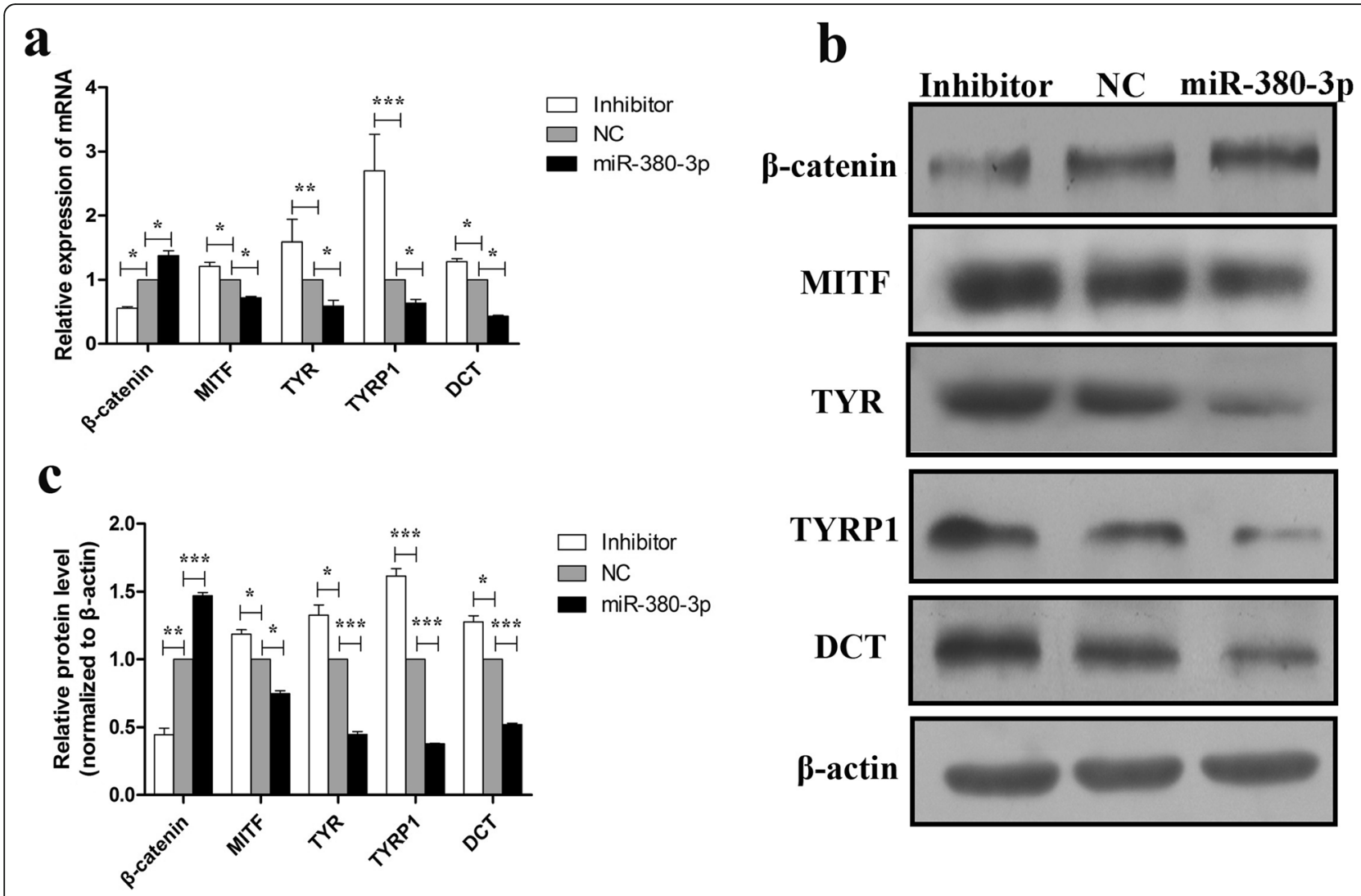

Fig. 4 Effect of miR-380-3p on mRNA and protein expression levels of $\beta$-catenin and melanogenic genes. a RT-qPCR analysis of $\beta$-catenin, MITF, TYR, TYRP1, and DCT mRNA expression levels in melanocytes transfected with miR-380-3p and its inhibitor compared with those in negative control (NC) melanocytes. b, c Western blot and quantitative analysis of $\beta$-catenin, MITF, TYR, TYRP1, and DCT protein levels in melanocytes transfected with miR-380-3p and its inhibitor compared with those in NC melanocytes. mRNA and protein expression levels were normalized to $18 \mathrm{~S}$ rRNA and $\beta$-actin expression, respectively. The bars represent the mean \pm standard error $(n=3)$. ${ }^{*} P<0.05,{ }^{* *} P<0.01,{ }^{* * *} P<0.001$

380-3p probe sequence was 5'-GTGGACCATACTAC ATACGACACAGAAG-3'; the NC (scrambled sequence) was 5'-GTGTAACACGTCTATACGCCCA-3' (Bio-High Technology, Hebei City, China).

\section{Construction of plasmids}

The plasmid containing miR-380-3p was constructed by inserting pre-miR-380-3p oligonucleotides into the pcDNA6.2-GW/EmGFPmiR vector (Invitrogen, Carlsbad, CA, USA). The inhibitor plasmid was constructed in the same vector by inserting the antisense sequence of miR380-3p to obtain pcDNA6.2-GW/EmGFPmiR380-inhibitor. The NC plasmid was constructed using a scrambled sequence of miR-380-3p.

A luciferase reporter plasmid was constructed by inserting the 3 '-untranslated region (UTR) of the alpaca SOX6 gene, including the putative miR-380-3p-binding site, into a dual luciferase pmirGL0 vector (Promega, Madison, WI, USA). Primers containing SacI and XbaI sites were used to amplify the 3'-UTR of the alpaca SOX6 gene (Additional file 1: Table S1) using skin cDNA as a template. The PCR product and the pmirGL0 vector digested with $\mathrm{SacI}$ and $\mathrm{XbaI}$ were ligated at $22{ }^{\circ} \mathrm{C}$ to obtain pmirGL0-SOX6-wt using a T4 ligation kit (Takara, Dalian, China). Additionally, the binding sites of miR-380 targeting the 3'-UTR of SOX6 were mutated using a site-directed gene mutagenesis kit (Beyotime, Shanghai, China), which was ligated with pmirGL0 to construct pmirGL0-SOX6-mut. The alpaca SOX6 coding region was amplified by PCR from cDNA (primers are listed in Additional file 1: Table S1) and cloned into the pcDNA3.1 expression vector with $\mathrm{SacI}$ and $\mathrm{XbaI}$ restriction sites. SOX6 NC (scrambled) was obtained from Invitrogen (Carlsbad, CA, USA). SOX6-siRNA and NC siRNA were purchased from Sangon Biotech Co Ltd. (Shanghai, China). All constructs were confirmed by sequencing.

\section{Melanocyte transfection and total RNA and protein preparation}

Alpaca melanocytes $\left(10^{6}\right.$ cells $)$ were cultured in melanocyte medium (MelM) (ScienCell Research Laboratories, Carlsbad, CA, USA) supplemented with $1 \%$ melanocyte growth supplement (MelGS), 1\% penicillin-streptomycin and $0.5 \%$ fetal bovine serum in a 6 -well plate in an incubator at $37^{\circ} \mathrm{C}$ under an atmosphere of $5 \% \mathrm{CO}_{2}$. 
a

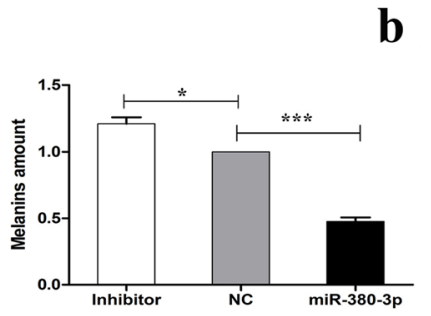

C

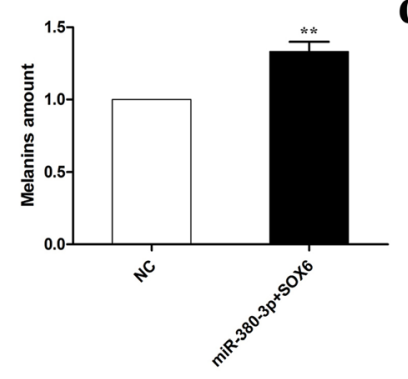

d

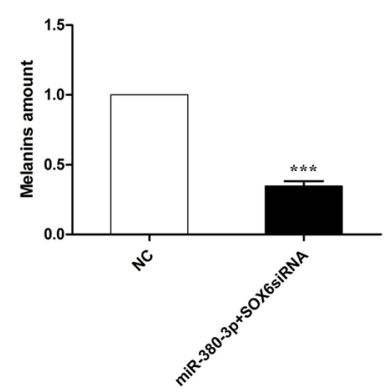

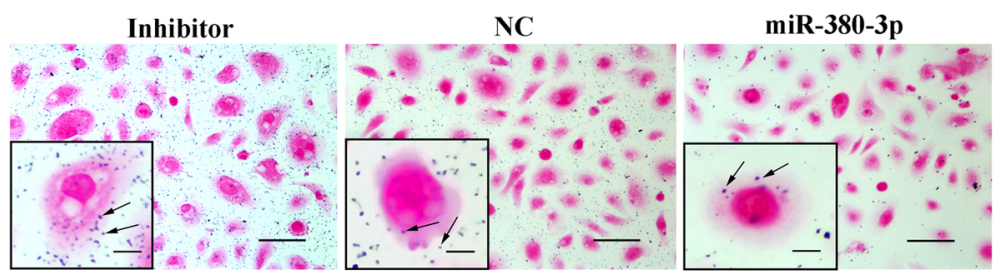

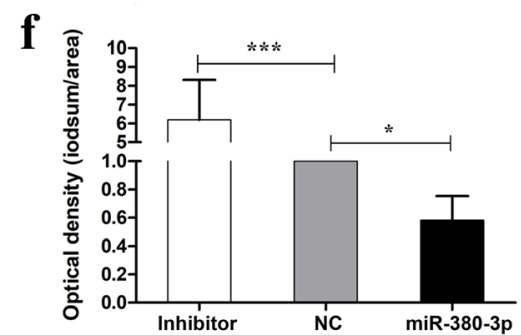

Fig. 5 Effect of miR-380-3p on melanin production in alpaca melanocytes. a Effect of miR-380-3p overexpression and knockdown on melanogenesis compared with negative control (NC) conditions. $\mathbf{b}$ Effect of miR-380-3p on cell pellets. $\mathbf{c}$ Effect of miR-380-3p overexpression and SOX6 on melanogenesis compared with negative control (NC) conditions. $\mathbf{d}$ Effect of miR-380-3p overexpression and SOX6 siRNA on melanogenesis compared with negative control (NC) conditions. e Effect of miR-380-3p overexpression and inhibition in melanocytes on melanin according to Fontana-Masson staining. $\mathbf{f}$ Quantitative analysis of the distribution of melanin particles. The bars represent the mean \pm standard error $(n=3)$. ${ }^{* *} P<0.001$

When the alpaca melanocytes reached approximately $80 \%$ confluence, they were transfected with pcDNA6.2GW/EmGFPmiR-380-3p, pcDNA6.2-GW/EmGFPmiR380inhibitor, pcDNA3.1-SOX6, SOX6-siRNA or the NC using Lipofectamine 2000 (Invitrogen, Carlsbad, CA, USA) according to the manufacturer's guidelines. Melanocytes were collected $72 \mathrm{~h}$ after transfection, washed 3 times with PBS and divided into two parts for protein and total RNA preparation. For total RNA preparation, $600 \mu \mathrm{l}$ Trizol (Invitrogen, Carlsbad, CA, USA) was added. After mixing, chloroform was added at a 1:0.2 ratio, mixed vigorously for $30 \mathrm{~s}$, and allowed to stand on ice for $3 \mathrm{~min}$. Then, the samples were centrifuged at $12,000 \mathrm{rpm}$ for $15 \mathrm{~min}$ at $4{ }^{\circ} \mathrm{C}$. The aqueous phase was removed, and isopropanol was added at a 1:2 ratio. The samples were then centrifuged at 12,000 rpm for $10 \mathrm{~min}$ at $4{ }^{\circ} \mathrm{C}$ to obtain pellets; $1 \mathrm{ml}$ of $75 \%$ icecold ethanol was added to the pellets and centrifuged at $5^{\circ} \mathrm{C}$ for $5 \mathrm{~min}$ at $4{ }^{\circ} \mathrm{C}$. Finally, $50 \mu \mathrm{l}$ of diethyl pyrocarbonate (DEPC) water was added to the RNA, which was treated with DNase I (Sigma, St. Louis, MO, USA). For protein preparation, $300 \mu \mathrm{l}$ of protein lysate and $3 \mu \mathrm{l}$ of phenylmethylsulfonyl fluoride (PMSF) were added and allowed to stand on ice for $30 \mathrm{~min}$. After centrifugation at 12,000 rpm for $10 \mathrm{~min}$ at $4{ }^{\circ} \mathrm{C}$, the supernatants were taken to obtain proteins. The RNA integrity was assessed by gel electrophoresis (Additional file 3: Figure S1), and the 


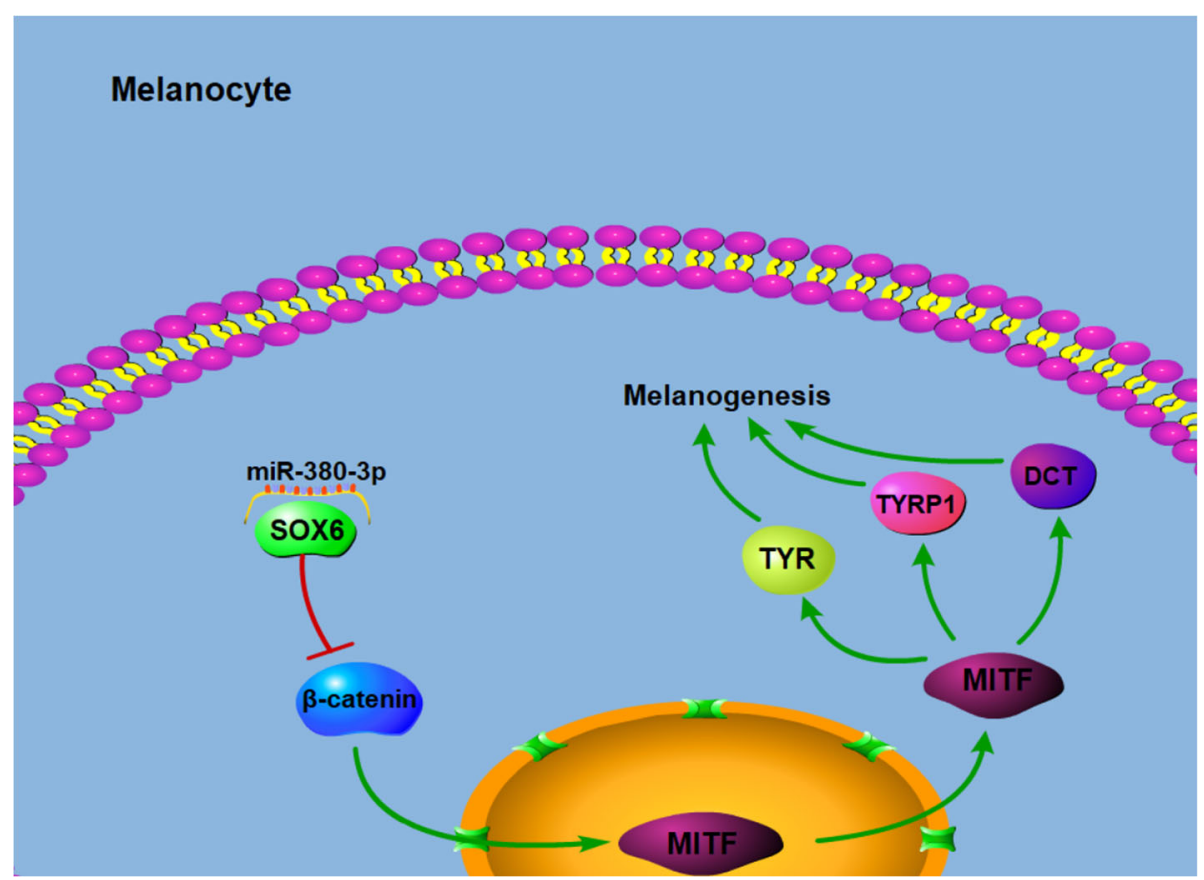

Fig. 6 Schematic representation of a potential pathway through which miR-380-3p may regulate melanogenesis based on the results of this study

concentrations were measured using a NanaDrop 1000 spectrophotometer (NanoDrop, Wilmington, NA, USA) (Additional file 2: Table S2). The protein concentrations were measured by the bicinchoninic acid (BCA) method.

\section{Dual luciferase assay}

For miRNA target validation, $3 \mu \mathrm{g}$ of pmirGL0-SOX6-wt, pmirGL0-SOX6-mut, pcDNA6.2-miR380-inhibitor and NC were cotransfected into 293T cells using Lipofectamine 2000. Luciferase activities in the transfected cells were measured with a dual luciferase reporter assay kit (Promega) $48 \mathrm{~h}$ after cotransfection according to the manufacturer's instructions. Firefly luciferase activity was normalized to Renilla luciferase activity, and the data are expressed as the relative luciferase activity (mean \pm standard error (SE), $n=3$ ).

\section{RT-qPCR assay for miRNA and mRNA expression}

For mRNA quantification, $1 \mu \mathrm{g}$ of total RNA from skin samples from 3 alpacas for each group with white, brown and black hair colors was converted to cDNA using a PrimeScript $^{\text {tw }}$ RT reagent kit with gDNA eraser (Takara Code No. RR047A, Dalian, China) according to the manufacturer's instructions; RT-qPCR was performed using TB Green ${ }^{\circ}$ Premix Ex Taq ${ }^{\text {Ta }}$ II (Takara Code No. RR820A, Dalian, China) following the MIQE guidelines. For miR-380-3p quantification, $1 \mu \mathrm{g}$ of total RNA from skin samples from 3 alpacas for each group with white, brown and black hair colors and melanocytes was converted to cDNA using Mir- $\mathrm{X}^{\mathrm{Tx}}$ miRNA first-strand synthesis and TB Green ${ }^{\text {TM }}$ qRT-PCR kits
(Clontech Code No. 638313, Dalian, China) according to the manufacturer's instructions and a specific stem-loop RT primer and common reverse primer according to a previously established method for real-time miRNA quantification [41]. RT-qPCR was then performed using the primer sequences listed in Additional file 1: Table S1 and TB Green ${ }^{\circ}$ Premix Ex Taq ${ }^{\text {Tw }}$ II (Takara Code No. RR820A) in a 7500 fast realtime PCR system (Applied Biosystems, Foster City, CA, USA). The thermal profile was $95^{\circ} \mathrm{C}$ for $10 \mathrm{~min}, 95^{\circ} \mathrm{C}$ for $30 \mathrm{~s}, 60^{\circ} \mathrm{C}$ for $30 \mathrm{~s}$, and $72^{\circ} \mathrm{C}$ for $20 \mathrm{~s}$ for 35 cycles. The biological and technical replicates were run in triplicate with melting curves to validate the amplification specificity.

The relative amounts of miRNAs and mRNAs were normalized to the amounts of U6 and $18 \mathrm{~s}$ rRNAs, respectively, based on the amplification efficiency test for the target and housekeeping genes. A sample without reverse transcriptase in the reaction was used as a NC. Total RNA from melanocytes transfected with the $\mathrm{NC}$ plasmid was used as a calibrator. The miRNA and mRNA transcript abundance was calculated using the comparative threshold cycle $\left(C_{T}\right)$ method [42] $(n=3$, mean \pm SE). The primer sequences are listed in Additional file 1: Table S1.

\section{Western blot analysis}

Proteins from skin and melanocyte samples were separated with $10 \%$ or $8 \%$ sodium dodecyl sulfate-polyacrylamide gel by electrophoresis, followed by transfer to polyvinylidene difluoride membranes. The membranes were blocked with $5 \%$ skim milk for $2 \mathrm{~h}$ and incubated with the following 
primary antibodies overnight at $4{ }^{\circ} \mathrm{C}$ : polyclonal rabbit antiSOX6 antibody at a 1:1000 dilution (Abcam, Cambridge, MA, USA), polyclonal rabbit anti- $\beta$-catenin antibody at a 1: 1000 dilution (Abcam), mouse anti-MITF at a 1:1000 dilution (Thermo, Waltham, MA, USA), polyclonal rabbit antiTYR antibody at a 1:1000 dilution (Abcam), polyclonal rabbit anti-TYRP1 antibody at a 1:1000 dilution (Abcam), and rabbit anti-DCT at a 1:1000 dilution (Abcam). The membranes were washed three times with Tris-buffered saline Tween-20 (TBST) (10 min/wash) and incubated for $1 \mathrm{~h}$ at $37^{\circ} \mathrm{C}$ with horseradish peroxidase-conjugated secondary antibodies against rabbit or mouse IgG at a 1:10000 dilution (Sigma). The membranes were washed four times with TBST ( $5 \mathrm{~min} /$ wash), and bound antibodies were visualized using enhanced chemiluminescence. Immunoblots were scanned on a ChemiDOC XRS+ imager (Bio-Rad Laboratories, Philadelphia, PA, USA). Protein levels were quantified using Image-Pro Plus software (Olympus, Japan).

\section{Melanin content measurement}

Melanocytes transfected with pcDNA6.2-GW/EmGFPmiR-380-3p, pcDNA6.2-GW/EmGFPmiR380-inhibitor, pcDNA3.1-SOX6, SOX6-siRNA or NC plasmid were harvested and rinsed with PBS, followed by the addition of $1 \mathrm{ml}$ of $1 \mathrm{M} \mathrm{NaOH}$ to dissolve melanin. The content of alkali-soluble melanin in the supernatant was measured by a spectrophotometer (Molecular Devices, Sunnyvale, CA, USA) at an absorbance of $475 \mathrm{~nm}$ and normalized to the total number of melanocytes. All experiments were performed in triplicate.

\section{Masson-Fontana staining for melanins}

After transfection was terminated, the cells on slides were washed 3 times with PBS for 3 min each time and fixed with $4 \%$ paraformaldehyde for $20 \mathrm{~min}$. Then, the slides were treated with a silver ammonia solution and placed in an oven at $60^{\circ} \mathrm{C}$ for $45 \mathrm{~min}$. Next, the slides were treated with a gold chloride solution $(0.2 \%)$ and sodium thiosulphate solution (35.6\%) at room temperature for $2 \mathrm{~min}$. A nuclear solid red solution was used for staining, and the slides were sealed with a neutral gum. Three fields of view were selected randomly for each image, and image analysis was performed using Image-Pro Plus software (Olympus, Japan). Intracellular melanin staining was quantified as the integrated optical density (IOD) obtained from the combined area and gray value, and the mean value was calculated.

\section{Statistical analysis}

The data are presented as the mean \pm SE. The differences in miRNA, mRNA, and protein contents, relative luciferase activities, and melanin content between the NC and experimental groups were determined by analysis of variance and Fisher's least significant difference tests using SPSS 17.0 software (Chicago, IL, USA).

\section{Supplementary information}

Supplementary information accompanies this paper at https://doi.org/10. 1186/s12864-019-6343-4

Additional file 1: Table S1. Primers used in the study.

Additional file 2: Table S2. Quality of the RNAs.

Additional file 3: Figure S1. RNA quality testing

\begin{abstract}
Abbreviations
BCA: Bicinchoninic acid; CAMP: cyclic adenosine monophosphate; $C_{T:}$ :Threshold cycle; DCT: Dopachrome tautomerase; DEPC: Diethyl pyrocarbonate; FSHD: Facioscapulohumeral muscular dystrophy; IncRNA: long noncoding RNA; miRNA: microRNA; MITF: Microphthalmiaassociated transcription factor; MLPH: Melanophilin; NC: Negative control; PBS: Phosphate-buffered saline; PMSF: Phenylmethylsulfonyl fluoride; RISC: RNA-induced silencing complex; RT-qPCR: Quantitative real-time PCR; SGC: soluble guanylate cyclase; SOX6: Sex-determining region Y-box 6; TBST: Tris-buffered saline Tween-20; TYR: Tyrosinase; TYRP1: Tyrosine-related protein-1; TYRP2: Tyrosine-related protein-2; UTR: Untranslated region; Wnt1: Wnt family member 1
\end{abstract}

Acknowledgments

We would like to thank the anonymous reviewers for their constructive comments.

Authors' contributions

$X X L$ and RWF conceived and designed the experiments. XXL and BD performed most of the experiments. XXL, BD and RWF wrote the paper. PQZ, JZZ, ZWZ and $\mathrm{BL}$ analyzed the data. All authors read and approved the final manuscript.

\section{Funding}

The study was supported by funding from the Young Sanjin Scholars Distinguished Professor Program (to R.F.) and the Aid Program for Innovation Research Teams at Shanxi Agricultural University (CXTD201202). The funding agencies did not participate in the study design, sample collection, analysis, data interpretation or writing of this research.

Availability of data and materials

All data generated or analyzed during this study are included in this published article and its supplementary information files.

Ethics approval and consent to participate

All animals were handled and treated according to the alpaca guidelines approved by the Animal Ethics Committee [2017(55)] at Shanxi Agricultural University (Taigu, Shanxi, China).

Consent for publication

Not applicable.

Competing interests

The authors declare that they have no competing interests.

\section{Author details}

${ }^{1}$ College of Animal Science and Veterinary Medicine, Shanxi Agricultural University, Mingxian South Road, Taigu, China. ${ }^{2}$ Department of Ecology Research, Beijing Milu Ecological Research Center, Nanhaizi, Daxing district, Beijing, China. ${ }^{3}$ College of Life Science, Shanxi Agricultural University, Taigu, China.

Received: 6 January 2019 Accepted: 27 November 2019

Published online: 10 December 2019

\section{References}

1. Cecchi T, Valbonesi A, Passamonti P, Gonzale M, Antonini M, Renieri C. Quantitative variation of melanins in alpaca (Lama pacos L.). Ital J Anim Sci. 2011;10(3):e30.

2. Levy $C$, Khaled M, Fisher DE. MITF: master regulator of melanocyte development and melanoma oncogene. Trends Mol Med. 2006;12:406-14. 
3. Bernstein E, Caudy AA, Hammond SM, Hannon GJ. Role for a bidentate ribonuclease in the initiation step of RNA interference. Nature. 2001; 409(6818):363-6.

4. Shenoy A, Blelloch RH. Regulation of microRNA function in somatic stem cell proliferation and differentiation. Nat Rev Mol Cell Biol. 2014;15(9):565-76.

5. Mione M, Bosserhoff A. MicroRNAs in melanocyte and melanoma biology. Pigment Cell Melanoma Res. 2015;28(3):340-54.

6. Dong C, Wang H, Xue L, Dong Y, Yang L, Fan R, et al. Coat color determination by miR-137 mediate down-regulation of microphthalmiaassociated transcription factor in a mouse model. RNA. 2012;18:1679-86.

7. Wu D, Chen J, Chang D, Lin S. Mir-434-5p mediates skin whitening and lightening. Clin Cosmet Investig Dermatol. 2008;1:19-35.

8. Guo J, Zhang JF, Wang WM, et al. MicroRNA-218 inhibits melanogenesis by directly suppressing microphthalmia-associated transcription factor expression. RNA Biol. 2014;11(6):732-41.

9. Wang PC, Zhao YY, Fan RW, Chen TZ, Dong CS. MicroRNA-21a-5p Functions on the Regulation of Melanogenesis by Targeting Sox 5 in Mouse Skin Melanocytes. Int J Mol Sci. 2016;17(7):959.

10. Dynoodt P, Mestdagh P, Van Peer G, et al. Identification of miR-145 as a key regulator of the Pigmentary process. J Investig Dermatol. 2013;133(1):201-9.

11. Goswami S, Tarapore RS, TeSlaa JJ, Grinblat Y, Setaluri V, Spiegelman VS. MicroRNA-340-mediated degradation of Microphthalmia-associated transcription factor mRNA is inhibited by the coding region determinantbinding protein. J Biol Chem. 2010;285:20532-40.

12. Zhao $Y$, Wang $P$, Meng J, Ji $Y, X u$ D, Chen T, et al. MicroRNA-27a-3p inhibits Melanogenesis in mouse skin melanocytes by targeting Wnt3a. Int J Mol Sci. 2015;16(5):10921-33.

13. Zhang J, Liu Y, Zhu Z, Yang S, Ji K, Hu S, et al. Role of microRNA508-3p in melanogenesis by targeting microphthalmia transcription factor in melanocytes of alpaca. Animal. 2017;11(02):236-43.

14. Yang S, Liu B, Ji K, Fan R, Dong C. MicroRNA-5110 regulates pigmentation by cotargeting melanophilin and WNT family member 1. FASEB J. 2018; 32(10):5405-12.

15. Zhu Z, He J, Jia J, Bai R, Yu X, Lv L, et al. MicroRNA-25 functions in regulation of pigmentation by targeting the transcription factor MITF in alpaca (Lama pacos) skin melanocytes. Domest Anim Endocrinol. 2010;38(3):200-9.

16. Ji K, Zhang P, Zhang J, Fan R, Liu Y, Yang S, et al. MicroRNA 143-5p regulates alpaca melanocyte migration, proliferation and melanogenesis. Exp Dermatol. 2018;27(2):166-71.

17. Yang S, Fan R, Shi Z, Ji K, Zhang J, Wang H, et al. Identification of a novel microRNA important for melanogenesis in alpaca (Vicugna pacos). J Anim Sci. 2015;93(4):1622-31.

18. Gordon MW, Yan F, Zhong X, Mazumder PB, Xu-Monette ZY, Zou D, et al. Regulation of p53-targeting microRNAs by polycyclic aromatic hydrocarbons: implications in the etiology of multiple myeloma. Mol Carcinog. 2015;54(10):1060-9

19. Zhang $B$, Pan X. RDX induces microRNA aberrant expression in mouse brain and liver. Environ Health Perspect. 2009;117(2):231-40.

20. Warner DR, Mukhopadhyay P, Brock G, Webb CL, Michele PM, Greene RM. MicroRNA expression profiling of the developing murine upper lip. Develop Growth Differ. 2014;56(6):434-47.

21. Papaioannou MD, Pitetti JL, Ro S, Park C, Aubry F, Schaad O, et al. Sertoli cell Dicer is essential for spermatogenesis in mice. Dev Biol. 2009;326(1): 250-9.

22. Cheloufi S, Dos Santos CO, Chong MMW, Hannon GJ. A dicer-independent miRNA biogenesis pathway that requires ago catalysis. Nature. 2010; 465(7298):584-9.

23. Stolt CC, Schlierf A, Lommes P, Hillgärtner S, Werner T, Kosian T, et al. SoxD proteins influence multiple stages of oligodendrocyte development and modulate SoxE protein function. Dev Cell. 2006;11:697-709.

24. Lefebvre R. The SoxD transcription factors - Sox5, Sox6, and Sox13 - are key cell fate modulators. Int J Biochem Cell Biol. 2010:42(3):429-32.

25. Haldin CE, Labonne C. SoxE factors as multifunctional neural crest regulatory factors. Int J Biochem Cell Biol. 2010;42(3):441-4.

26. Leow SC, Poschmann J, Too PG, Yin J, Joseph R, McFarlane C, et al. The transcription factor SOX6 contributes to the developmental origins of obesity by promoting adipogenesis. Development. 2016;6:950.

27. Bellei B, Pitisci A, Catricalà C, Larue L, Picardo M. Wnt/B-catenin siging is stimulated by a-melanocyte-stimulating hormone in melanoma and melacyte cells: implication in cell differentiation. Pigment Cell Melanoma Res. 2011;24(2):309-25
28. Du B, Liu X, Zhang P, Ji K, Hu S, Yang S, et al. The role of sex-determining region Y-box 6 in melanogenesis in alpaca melanocytes. J Dermatol Sci. 2018;91(3):268-75

29. Dong CS, Yang SS, Fan RW, Ji KY, Zhang JZ, Liu XX, et al. Functional role of Cyclin-dependent kinase 5 in the regulation of Melanogenesis and epidermal structure. Sci Rep. 2017;7(1):13783.

30. Iguchi H, Urashima Y, Inagaki Y, Ikeda Y, Okamura M, Tanaka T, et al. Sox6 suppresses CyclinD1 promoter activity by interacting with beta-catenin and histone deacetylase 1, and its down-regulation induces pancreatic beta-cell proliferation. J Biol Chem. 2007;282(26):19052-61.

31. Lin JY, Fisher DE. Melanocyte biology and skin pigmentation. Nature. 2007; 445(7130):843-50.

32. Zehavi L, Avraham R, Barzilai A, Bar-Ilan D, Navon R, Sidi $Y$, et al. Silencing a large microRNA cluster on human chromosome 14q32 in melanoma: biological effects of mir-376a and mir-376c on insulin growth factor 1 receptor. Mol Cancer. 2012;11(1):44.

33. Iwakawa H, Tomari Y. The functions of MicroRNAs: mRNA decay and translational repression. Trends Cell Biol. 2015;25(11):651-65.

34. Damsky WE, Curley DP, Santhanakrishnan M, Rosenbaum LE, Platt JE, Gould Rothberg BE, et al. Gbeta-catenin signaling controls metastasis in Brafactivated Pten-deficient melanomas. Cancer Cell. 2011;20(6):741-54.

35. Widlund HR, Horstmann MA, Price ER, Cui J, Lessnick SL, Wu M, et al. $\beta$ Catenin-induced melanoma growth requires the downstream target Microphthalmia-associated transcription factor. J Cell Biol. 2002;158(6):1079-87.

36. Vachtenheim J, Borovanský J. "Transcription physiology" of pigment formation in melanocytes: central role of MITF. Exp Dermatol. 2010;19(7): 617-27.

37. Schmidt C, Patel K. Wnts and the neural crest. Anat Embryol. 2005;209(5): 349-55.

38. Elworthy S, Lister JA, Carney TJ, Raible DW, Kelsh RN. Transcriptional regula tion of mitfa accounts for the Sox10 requirement in zebrafish melanophore development. Development. 2003;130:2809-18.

39. Bentley NJ, Eisen T, Goding CR. Melanocyte-specific expression of the human tyrosinase promoter: activation by the microphthalmia gene product and role of the initiator. Mol Cell Biol. 1994;14(12):7996-8006.

40. Bai R, Sen A, Yu Z, Yang G, Fan R, Lv L, et al. Validation of methods for isolation and culture of alpaca melanocytes: a novel tool for in vitro studies of mechanisms controlling coat color. Asian Aust J Anim Sci. 2010;23(4): 430-6.

41. Chen C, Ridzon DA, Broomer AJ, Zhou Z, Lee DH, Nguyen JT, et al. Realtime quantification of microRNAs by stem-loop RT-PCR. Nucleic Acids Res. 2005;33(20):e179.

42. Livak KJ, Schmittgen TD. Analysis of relative gene expression data using real-time quantitative PCR and the 2(-Delta Delta C(T)) method. Methods. 2001;25(4):402-8.

\section{Publisher's Note}

Springer Nature remains neutral with regard to jurisdictional claims in published maps and institutional affiliations.
Ready to submit your research? Choose BMC and benefit from:

- fast, convenient online submission

- thorough peer review by experienced researchers in your field

- rapid publication on acceptance

- support for research data, including large and complex data types

- gold Open Access which fosters wider collaboration and increased citations

- maximum visibility for your research: over $100 \mathrm{M}$ website views per year

At $\mathrm{BMC}$, research is always in progress.

Learn more biomedcentral.com/submission 